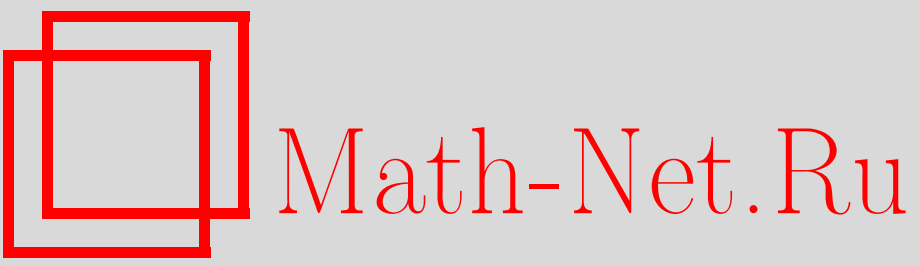

Э. Б. Винберг, И. М. Гельфанд, С. Г. Гиндикин, Е. Б. Дынкин, В. А. Малышев, Р. А. Минлос, А. Л. Онищик, И. И. Пятецкий-Шапиро, А. Н. Рыбко, Ю. М. Сухов, С. Б. Шлосман, Фридрих Израилевич Карпелевич (некролог), УМН, 2001, том 56, выпуск 1, 147-152

DOI: https://doi.org/10.4213/rm359

Использование Общероссийского математического портала Math-Net.Ru подразумевает, что вы прочитали и согласны с пользовательским соглашением

http://www. mathnet.ru/rus/agreement

Параметры загрузки:

IP: 54.89 .56 .158

26 апреля 2023 г., 14:09:49 


\section{ФРИДРИХ ИЗРАИЛЕВИЧ КАРПЕЛЕВИЧ}

5 июля 2000 года ушел из жизни замечательный московский математик профессор кафедры прикладной математики Московского государственного университета путей сообщения, ведущий научный сотрудник Добрушинской математической лаборатории Института проблем передачи информации РАН Фридрих Израилевич Карпелевич.

Ф.И. Карпелевич родился 2 октября 1927 года в Москве. С 1947 по 1952 год он был студентом механико-математического факультета Московского государственного университета им. М.В. Ломоносова. В связи с проводившейся в это время политикой государственного антисемитизма, несмотря на блестящую учебу и очевидный талант, $\Phi$ ридрих Израилевич не был оставлен в аспирантуре мех-мата, а был отправлен по распределению преподавать в техникуме в Новочеркасске, где он проработал один год. После этого он серьезно заболел, и ему разрешили покинуть место распределения и вернуться домой в Москву.

Оказавшись в Москве, он случайно встретил на улице профессора П.К. Рашевского, которьй помог ему устроиться на работу в Московский институт инженеров транспорта на факультет прикладной математики, где Фридрих Израилевич проработал всю свою жизнь, начиная с 1953 года, а с 1968 по 1998 год заведовал кафедрой прикладной математики. За его научные и педагогические заслуги $\Phi$. И. Карпелевич был удостоен звания "Заслуженный деятель науки”.

Научным руководителем $\Phi$. И. Карпелевича в Московском университете был Евгений Борисович Дынкин, знавший его со школьных лет: в 1944-45 гг. Фридрих Карпелевич был одним из самых активных участников школьного математического кружка при Московском университете, которьп руководил Е.Б. Дынкин (сам бывший в то время студентом).

$\Phi$. И. Карпелевич получил первые результаты еще в ранние студенческие годы. Широко известна его работа 1949 г. о характеристических корнях матриц с неотрицательными элементами [2]. Он дал окончательное решение проблемы А. Н. Колмогорова, в которой незадолго до этого существенньй прогресс был достигнут Н. А. Дмитриевым и Е. Б. Дынкиным. Заметим, что эта работа была уже второй публикацией студента Карпелевича. Его работа [1] была опубликована в Успехах математических наук, когда он был студентом первого курса.

С начала 50-х годов $\Phi$. И. Карпелевич исследует подалгебры в полупростых алгебрах Ли. Он начинает с простого единообразного описания неполупростых максимальных подалгебр простых комплексных алгебр Ли, которые были ранее классифицированы В.В.Морозовым. В этой работе был введен класс подалгебр, назьваемых в настоящее время параболическими, и дано их хорошо известное описание в терминах систем простых корней или схем Дынкина. Следующие пять лет он изучает полупростые подалгебры вещественных полупростых алгебр Ли. Незадолго до этого Е. Б. Дынкин описал полупростые подалгебры комплексных полупростых алгебр Ли. Переход к вещественному случаю потребовал принципиально новых соображений. Одним из них была знаменитая теорема Карпелевича о каноническом вложении вещественной полупростой 
подалгебры Ли. Для ее доказательства он применил геометрию симметрических пространств, которые впоследствии стали его любимым математическим объектом. Далее, он получил замечательную формулу для индексов инерции инвариантной симметрической или эрмитовой формы в пространстве неприводимого линейного представления вещественной полупростой алгебры Ли, и это практически дало полное решение задачи для классических алгебр Ли.

В 1956 году за свои работы он получил премию Московского математического общества, которой всю жизнь гордился.

С конца 50-х годов интересы $Ф$. И. Карпелевича сдвигаются с алгебраических проблем теории групп и алгебр Ли к проблемам геометрии однородных многообразий и анализа на них. Отметим его работу 1958 г. с $\Phi$. А. Березиным [14], в которой они выразили зональные сфферические функции на грассманианах через специальные функции от одного переменного. Зональные сферические функции на римановых симметрических пространствах - один из важнейших классов специальных функций от нескольких переменных. Они являются функциями от одного переменного для пространств ранга 1 и в этом случае выражаются через гипергеометрическую функцию Гаусса. И.М. Гельфанд и М. А. Наймарк вычислили их в случае комплексных групп движений. Многочисленные попытки провести вычисления в других случаях оказались безуспешными, и вьгисления Березина-Карпелевича остаются уникальными и по сей день.

В эти годы Хариш-Чандра начал изучать асимптотику зональных сферических функций. Он дал интегральное представление для коэффициента в асимптотике, которое стали называть c-функцией Хариш-Чандры. Через эту функцию просто выражается плотность в формуле Планшереля. В 1962 году С. Г. Гиндикин и Ф. И. Карпелевич [18] явно вычислили $c$-функцию как произведение В-функций и, как следствие, получили явное выражение для плотности в формуле Планшереля. Этот результат широко известен сегодня как формула Гиндикина-Карпелевича. В дальнейших работах эта формула была связана с функциональньми уравнениями для сплетающих операторов и была применена к обращению орисферического преобразования на римановых симметрических пространствах.

Одним из наиболее выдающихся достижений $\Phi$. И. Карпелевича в теории симметрических пространств является его конструкция границы римановых симметрических пространств неположительной кривизны, полученная в 1965 году [25]. В ее основе - глубокое изучение асимптотического поведения геодезических. Граница Карпелевича имеет многочисленные применения, начиная с граничных задач для собственных функций оператора Лапласа-Бельтрами. Он еще продолжил изучение этого оператора, прежде чем окончательно изменить предмет своих исследований и заняться задачами теории вероятностей.

Интерес к вопросам теории вероятностей у $\Phi$. И. Карпелевича возник под влиянием Е. Б. Дьнкина: в 50-60-е годы Фридрих Израилевич с энтузиазмом участвовал в работе дынкинского семинара по теории марковских процессов. Круг вопросов, которыми он интересовался, был достаточно широк, хотя в большинстве случаев его результаты имели тесную связь с теорией представлений, особенно с ее геометрическими аспектами. Ярким примером такого рода служит работа [15]. Здесь было впервые дано определение гауссовского распределения на пространстве Лобачевского и сформулированы теорема о характеристической функции свертки распределений, а также центральная предельная теорема для пространства Лобачевского. В последние годы он вернулся к этой тематике: в работе [79] была вычислена хаусдорфова размерность случайного множества предельных точек для однородного ветвящегося процесса на пространстве Лобачевского любой размерности, а в [80] найдена элегантная явная формула для функции больших уклонений для однородного случайного блуждания на трехмерном пространстве Лобачевского.

С 1975 года Ф.И. Карпелевич активно занимался задачами прикладной теории вероятностей, в частности, теорией очередей. Его стиль и методы были основаны на блестящей аналитической технике, что позволило ему достичь чрезвычайно тонких результатов. Вместе с тем он обладал великолепной интуицией, что делало его работы интересными для широкого круга прикладных специалистов, особенно в современной теории сетей с очередями.

Работы Ф. И. Карпелевича по теории очередей включают серию статей [41], [44], [59], [62], [76] и монографию [69], написанные совместно с его учеником А. Я. Крейниным, по теории многофазных систем, где заявки последовательно обслуживаются цепочкой серверов. В 1993-1998 годах вместе с М. Я. Кельбертом и Ю. М. Суховым был написан другой цикл работ [63]-[67], [75], [77], инициированный изучением сетей с синхронизацией. Это направлениепринесло много неожиданных результатов, в том числе, в задачах, напрямую не связанных с сетевой тематикой. Особую роль сыграла статья [74], вызвавшая повьшенный интерес в мировой литературе. В ней на прос- 
том и красивом примере было показано, что элементы локального управления на сети приводят к впечатляющему улучшению ее параметров.

В развитие этой тематики Ф.И. Карпелевич выполнил в последнее время ряд работ с А.Н. Рыбко [81]-[83]. В этих работах исследовался термодинамический предельный переход для моделей среднего поля, описьвающих работу сетей массового обслуживания. В них впервые был развит математический аппарат, при помощи которого была доказана для довольно общей ситуации сходимость соответствующих случайных процессов к предельньп динамическим системам.

В последние годы Карпелевич совместно с В.А. Мальшевым и А. Н. Рыбко сделал пионерскую работу [72], в которой впервые математически строго при помощи эйлеровского скейлинга исследовались нейронные сети биологической природы. Отметим также, что остались еще неопубликованными некоторые работы, открьвающие новые перспективы, в которых $Ф$ ридрих Израилевич принимал участие.

Для многих коллег Фридриха Израилевича даже более важньм, чем его выдающийся талант математика, являлась его ангельская доброта и удивительное внутреннее благородство его души. Его мнение, всегда справедливое и доброжелательное, было чрезвычайно важно для его коллег и учеников.

Э.Б. Винберг, И.М. Гельфанд, С.Г. Гиндикин, Е. Б. Дынкин, В.А. Мальишев, Р.А. Минлос, А. Л. Онищик, И. И. Пятецкий-Шапиро, А. Н. Рьбко, Ю. М. Сухов, С. Б. Шлосман

\section{СПИСОК НАУ ЧНЫХ РАБОТ Ф. И. КАРПЕЛЕВИЧА}

[1] Псевдонормы в кольце целых чисел // УМН. 1948. Т. 3. № 5. С. 174-177

[2] О характеристических корнях матриц с неотрицательными коэффиициентами // УМН. 1949. T. 4. № 5. C. $177-178$

[3] О характеристических корнях матриц с неотрицательными элементами // Изв. АН СССР. Сер. матем. 1951. Т. 15. С. 361-383

[4] О неполупростых максимальных подалгебрах полупростых алгебр Ли // Докл. АН СССР. 1951. Т. 76. № 6. С. $775-778$

[5] Классификация простых подгрупп вещественных форм группы комплексных унимодулярных матриц // Докл. АН СССР. 1952. Т. 85. №6. С. 1205-1208

[6] Подгруппы вещественных групп Ли // УМН. 1952. Т. 7. № 5. С. 203-204

[7] Поверхности транзитивности полупростой подгруппы группы движений симметрического пространства // Докл. АН СССР. 1953. Т. 93. № 3. С. 401-404

[8] Классификация простых подалгебр вещественных форм классических алгебр // Докл. АН СССР. 1953. Т. 93. № 4. С. 613-616

[9] Простые подалгебры вешественных алгебр Ли // Труды ММО. 1955. Т. 4. С. 3-112

[10] О полупростых подгруппах полупростых групп Ли // УМН. 1955. Т. 10. № 1. С. 196

[11] Эрмитовы и билинейные инварианты подалгебр алгебры матриц // УМН. 1955. Т. 10 . № 4. C. $190-191$

[12] Алгебра гомологий пространства путей // Докл. АН СССР. 1956. Т. 106. №6. С. 967-969 (совм. с А.Л. Онишик)

[13] О расслоении однородных пространств // УМН. 1956. Т. 11. № 3. С. 131-138

[14] Зональные сферические функции и операторы Лапласа на некоторых симметрических пространствах // Докл. АН СССР. 1958. Т. 118. № 1. С. 9-12 (совм. с Ф. А. Березиньп)

[15] Предельные теоремы для композиций распределений в плоскости и пространстве Лобачевского / / Теория вероятн. и ее примен. 1959. Т. 4. № 4. С. 432-436 (совм. с В. Н. Тутубалиным, М. Г. Шуром)

[16] Геодезические линии и гармонические функции на симметрических пространствах // Докл. AH CCCP. 1959. T. 124. №6. C. 1199-1202

[17] Орисферические радиальные части оператора Лапласа на симметрических пространствах // Докл. АН СССР. 1962. Т. 143. № 9. С. 1034-1037

[18] Мера Планшереля для римановых симметрических пространств неположителњной кривизны // Докл. АН СССР. 1962. Т. 145. № 2. С. 252-255 (совм. с С. Г. Гиндикиным) 
[19] Элементы линейной алгебры и линейного программирования. М.: Физматгиз, 1963 (совм. с Л.Е. Садовским)

[20] Неотрицательные собственные функции оператора Белтрами-Лапласа на симметрических пространствах неположительной кривизны // Докл. АН СССР. 1963. Т. 151. № 6 . C. $1274-1276$

[21] Теория представлений групп Ли // Труды IV Всесоюзного математического съезда.. Т. 11, 1964. С. 275-281 (совм. с М. Граевым, А. Кирилловым)

[22] Об одной задаче интегральной геометрии // Юбилейный сборник памяти Н. Г. Чеботарева. Казань: Изд-во Казан. ун-та, 1964. С. 30-43 (совм. с С. Г. Гиндикиным)

[23] Элементы линейной алгебры и линейного программирования. 2-е изд. М.: Наука, 1965 (совм. с Л.Е. Садовским)

[24] Элементы линейной алгебры и линейного программирования. Пер. на япон. Токио, 1965 (совм. с Л.Е. Садовским)

[25] Геометрия геодезических и собственные функции оператора Бельтрами-Лапласа на симметрических пространствах // Труды ММО. 1965. Т. 14. С. 48-185

[26] О некоторых специальных функциях от нескольких переменных, связанных с группами Ли // Исследования по современным проблемам конструктивной теории функций. Баку: АН АзербССР, 1965. С. 545-554 (совм. с С. Г. Гиндикиным)

[27] Об одном интеграле, связанном с симметрическими римановыми пространствами неположительной кривизны // Изв. АН СССР. Сер. матем. 1966. Т. 30. № 5. С. 1148-1156

[28] Элементы линейной алгебры и линейного программирования. 3-е изд., испр. и доп. М.: Наука 1967 (совм. с Л.Е. Садовским)

[29] О представлениях алгебр Ли с дополненной структурой // Матем. сб. 1968. Т. 77. № 2 (совм. с $\Phi$. А. Березиньмм

[30] On an integral connected with symmetric Rieman species of nonpositive curvature // Amer. Math. Soc. Transl. 1969. V. 85. P. 249-257 (with S. Gindikin)

[31] Об одной минимаксной задаче оптимального управления // Труды МИИТ. 1970. № 310

[32] Об одном методе сведения многоцелевых задач линейного программирования к одноцелевым / / Опыт применения прикладных методов математики и вычислительной техники в народном хозяйстве. М.: Статистика, 1974 (совм. с В.А. Мухиной)

[33] О некоторых методах решения многоцелевых задач // Экономика и матем. методы. 1975. № 2 (совм. с В. А. Мухиной)

[34] Об одном методе решения многоцелевых задач линейного программирования // Автоматика и телемеханика. 1975. № 7. С. 153-155 (совм. с В. А. Мухиной)

[35] Современные методы расчета оптимального управления и перспективы их применения при проектировании приводов (электроприводов) // 8-я Всесоюзная научно-техническая конференция по автоматизированному электроприводу: тезисы докладов. М., 1975 (совм. с С.В. Страховым)

[36] Об ассоциативных алгебрах функций // Вестник МГУ. Сер. Матем., мех. 1976. Т. 31. № 1. С. $33-38$ (совм. с $\Phi$.А. Березиным)

[37] О приближении функций многих нормально-распределенных случайных переменных / Труды МИИТ. 1976. № 524 (совм. с В. К. Роговым)

[38] Оценки среднего времени ожидания в одноканальной системе $G I / G / 1 / /$ Изв. АН СССР. Сер. техн. киберн. 1976. Т. 3. С. 105-107 (совм. с А. Я. Крейниным)

[39] Оценка среднего времени ожидания в одноканальной системе массового обслуживания и приложения к решению некоторых задач // Труды МИИТ. 1977. № 549 (совм. с А. Я. Крейниным)

[40] Bounds for the mean waiting time for single server system GI/G/1// Cyb. 1976. V. 3 (with A. Ya. Kreinin)

[41] Some bounds for the mean waiting time in $E_{k} / G I / 1$ queues // Math. Operations Forsch. Statist., Ser. Statistics. 1980. V. 11. № 1. P. 85-88 (with A. Ya. Kreinin)

[42] Двухфазовая система массового обслуживания в режиме большой загрузки // Теория массового обслуживания: труды семинара. М.: ВНИИ системных исследований, 1981. С. 160-164 (совм. с А. Я. Крейниным)

[43] Двумерные асимптотические задачи теории массового обслуживания // Третья международная Вильнюсская конференция по теории вероятностей и математической статистике: тезисы докладов. Т. 1, 1981. С. 225 
[44] Двухфазовая система массового обслуживания $\left(G I / G / 1 \rightarrow G^{\prime} / 1 / \infty\right)$ в режиме большой загрузки // Теория вероятн. и ее примен. 1981. Т. 26. № 2. С. 302-320 (совм. с А. Я. Крейниным)

[45] One problem of integral geometry // Selecta Math. Soviet. 1981. V. 1. № 2. P. 169-184 (with S. G. Gindikin)

[46] Некоторые характеристики двухфазовых систем обслуживания // Автоматизированные системы массового обслуживания: тезисы докладов на Всесоюзном совещании, Нальчик, 1982. М., 1982. С. 174-175 (совм. с А. Я. Крейниным)

[47] Heavy traffic limit theorems for many-stagy queues // Fundamentals of Teletraffic Theory: Proceedings of the Third International Seminar of Teletraffic Theory. Moscow, 1984. P. 214-221 (with A. Ya. Kreinin)

[48] Характеристики двуфазовой системы $M / M / I M / I$ // IV Международная Вильнюсская конференция по теории вероятностей и математической статистике: тезисы докладов. Вильнюс, 1985. С. 21-22 (совм. с А. Я. Крейниным)

[49] Некоторые характеристики двухфозных систем // Изв. АН СССР. Сер. техн. киберн. 1985. № 2. С. 182-184 (совм. с А. Я. Крейниным)

[50] Показатели функционирования стационарных систем обслуживания в условиях высоких загрузок обслуживающих устройств // Доклад на XXII Общесетевой научно-технической конференции "Совершенствование эксплуатационной работы железных дорог с применением математических методов и ЭВМ”. Программа конференции. М., 1985 (совм. с И. Б. Сотниковьгм)

[51] О прогнозировании значений некоторых плановых показателей развития АПК. Всесоюзное научно-техническое совещание "Проблемы внедрения кибернетики в сельскохозяйственном производстве": тезисы совещания. Ереван 1986. С. 1 (совм. с В. А. Мухиной, Ю. В. Степановым)

[52] Предельные теоремы для многофазовых систем массового обслуживания // Вероятностные распределения и математическая статистика. Ташкент: $Ф А Н, ~ 1986$. С. 212-228 (совм. с А. Я. Крейниным)

[53] Предельные теоремы для многофазовых “телеграффных" систем массового обслуживания // Международный семинар по проблемам устойчивости стохастических моделей. Программа семинара. Сухуми, 1987 (совм. с А.Я. Крейниным)

[54] Очередность подачи-уборки вагонов // Вестник ВНИИЖТ. 1988. №1. С. 8-11 (совм. с И. Б. Сотниковым)

[55] K теории возбуждения колебаний кольцевой антенной вблизи нижнегибридного резонанса // Препринт № 1386 ИКИ, 1988 (совм. с И. Г. Соловьевьм, И.Б. Сотниковым, В. И. Шевченко, В. И. Карпманным)

[56] Показатели функционирования стационарных систем обслуживания в условиях высоких загрузок обслуживающих устройств // Межвузовский сборник научных трудов "Методы интенсификации процессов грузовой и коммерческой работы железных дорог”. № 791. М.: МИИТ, 1988. С. 34-36 (совм. с И. Б. Сотниковым)

[57] Структура электростатического поля кольцевой антенны с плоскостью витка, параллельной магнитному полю // Препринт № 1472 ИКИ, 1988 (совм. с В. А. Жаравиным, И. Б. Сотниковьм, В.И. Шевченко)

[58] On the characteristic roots of matrices with nonnegative elements // Amer. Math. Soc. Transl. Ser. 2. 1988. V. 140. P. 79-100

[59] Асимптотический анализ систем коммуникации сообщений телеграфного типа // Проблемы передачи информации. 1990. Т. 26. № 3. С. 83-95 (совм. с А. Я. Крейниным)

[60] A Queueing Network Model Leading to a Second-Orger Lingley Equation // Preprint № 617. Universität Heidelberg, 1991 (with M. Ya. Kelbert, Y.M. Suhov)

[61] The mean-field limit for a class of queueing networks // J. Statist. Phys. 1992. V. 6. № 3/4. P. 802-825 (with F. Baccelli, M. Ya. Kelbert, A. A. Pukhalskii, A. N. Rybko, Y. M. Suhov)

[62] Joint distributions in Poissonian tandem queues // Queueing Systems Theory Appl. 1992. V. 12. № 3/4. P. 273-286 (with A. Ya. Kreinin)

[63] Stochastic equations on random trees // Cellular Automata and Cooperative Behaviour / ed. E. Boccara et al. Dordrecht: Kluwer Acad. Publ., 1993. P. 323-342 (with M. Ya. Kelbert, Yu. M. Suhov) 
[64] The branching diffusion, stochastic equations and travelling wave solutions to the equation of Kolmogorov-Petrovskii-Piskunov // Cellular Automata and Cooperative Behaviour / ed. E. Boccara et al. Dordrecht: Kluwer Acad. Publ., 1993. P. 343-366 (with M. Ya. Kelbert, Yu. M. Suhov)

[65] Higher-order Lindley equations // Stochastic Process. Appl. 1994. V. 53. № 1. P. 65-96 (with M. Ya. Kelbert, Yu. M. Suhov)

[66] The boundedness of branching Markov processes // The Dynkin Festschrift. Markov Processes and Their Applications / ed. M. Freidlin. Boston: Birkhäuser, 1994. 143-152. (Progr. Probab. V. 34.) (with M. Ya. Kelbert, Yu. M. Suhov)

[67] Об оценке максимумов сумм случайных величин, индексированных ребрами графоов // Теория вероятн. и ее примен. 1994. Т. 39. № 4. С. 833-840

[68] Поллинг-системы с быстрым обслуживанием, интенсивным входящим потоком и постоянным временем переключения // Проблемы передачи информации. 1994. Т. 30. № 4. С. 45-58 (совм. с А. Я. Крейниным)

[69] Heavy Traffic Limit for Multi-Phase Queues. Providence, RI: Amer. Math. Soc., 1995. (Amer. Math. Soc. Transl. Ser. 2. V. 137.) (with A. Ya. Kreinin)

[70] On the work of E. B. Dynkin in the theory of Lie groups. Lie Groups and Lie Algebras: E. B. Dynkin's seminar // Amer. Math. Soc. Transl. Ser. 2. 1995. V. 169. P. 1-13 (with A. L. Onishchik, E. B. Vinberg)

[71] Домашниезадания по дисциплине "Теория вероятностей”. М.: МИИТ, 1995 (совм. с В. С. Куликовым)

[72] Stochastic evolution of neural networks // Markov Process. Relat. Fields. 1995. V. 1. № 1. P. 141-161 (with V. A. Malyshev, A. N. Rybko)

[73] Dobrushin's approach to queueing network theory // J. Appl. Math. Stochastic Anal. 1996. V. 9. № 4. P. 373-397 (with E. A. Pechersky, Yu. M. Suhov)

[74] Система обслуживания с выбором наименьшей из двух очередей - асимптотический подход // Проблемы передачи информации. 1996. Т. 32. № 1. С. 20-34 (совм. с Н. Д. Введенской, Р. Л. Добрушиным, Ф. И. Карпелевичем)

[75] Об ограниченности неоднородной ветвящейся диффузии на прямой // Докл. РАН. 1996. Т. 349. № 4. С. 449-450 (совм. с Ю. М. Суховым)

[76] Asymptotic analysis of queueing systems with identical service // J. Appl. Probab. 1996. V. 33. № 1. P. 267-281 (with A. Ya. Kreinin)

[77] A criterion of boundedness of discrete branching random walk / / Classical and Modern Branching Processes / ed. K. B. Athreya, P. Jagers. New York: Springer-Verlag, 1997. P. 141-156 (with Y.M. Suhov)

[78] Boundedness of one-dimensional branching Markov processes // J. Appl. Math. Stochastic Anal. 1997. V. 10. № 4. P. 307-332 (with Y. M. Suhov)

[79] A phase transition for hyperbolic branching processes // Comm. Math. Phys. 1998. V. 195. P. 627-642 (with E. A. Pecherskii, Y. M. Suhov)

[80] The Cramér transform and large deviations on the 3D Lobachevsky space // On Dobrushin's way. From Probability Theory to Statistical Physics. A volume in memory of R. L. Dobrushin / ed. R. A. Minlos, S. Shlosman, Y. M. Suhov. Providence, RI: Amer. Math. Soc., 2000. P. 117-131. (Amer. Math. Soc. Transl. Ser. 2. V. 198.) (with E. A. Pecherskii, Y.M. Suhov)

[81] Thermodynamical limit for symmetric closed queueing networks // On Dobrushin's way. From Probability Theory to Statistical Physics. A volume in memory of R. L. Dobrushin / ed. R. A. Minlos, S. Shlosman, Y. M. Suhov. Providence, RI: Amer. Math. Soc., 2000. P. 133-155. (Amer. Math. Soc. Transl. Ser. 2. V. 198.) (with A. N. Rybko)

[82] Thermodynamical limit for the mean field model of simple symmetrical closed queueing network // Markov Process. Relat. Fields. 2000. V. 6. №1. P. 89-105 (with A. N. Rybko)

[83] Асимптотическое поведение симметричной замкнутой сети массового обслуживания в термодинамическом пределе // Проблемы передачи инфформации. 2000. Т. 36 № 2. С. 1-27 (совм. с А. Н. Рыбко) 